Gut and Liver, Vol. 11, No. 4, July 2017, pp. 559-566

\title{
Comparison of Histologic Core Portions Acquired from a Core Biopsy Needle and a Conventional Needle in Solid Mass Lesions: A Prospective Randomized Trial
}

\author{
Ban Seok Lee ${ }^{1}$, Chang-Min Cho ${ }^{1}$, Min Kyu Jung ${ }^{1}$, Jung Sik Jang ${ }^{2}$, and Han Ik Bae ${ }^{2}$ \\ Departments of ${ }^{1}$ Internal Medicine and ${ }^{2}$ Pathology, Kyungpook National University Medical Center, Daegu, Korea
}

Background/Aims: The superiority of endoscopic ultrasound-guided fine needle biopsy (EUS-FNB) over EUS-guided fine needle aspiration (EUS-FNA) remains controversial. Given the lack of studies analyzing histologic specimens acquired from EUS-FNB or EUS-FNA, we compared the proportion of the histologic core obtained from both techniques. Methods: A total of 58 consecutive patients with solid mass lesions were enrolled and randomly assigned to the EUSFNA or EUS-FNB groups. The opposite needle was used after the failure of core tissue acquisition using the initial needle with up to three passes. Using computerized analyses of the scanned histologic slide, the overall area and the area of the histologic core portion in specimens obtained by the two techniques were compared. Results: No significant differences were identified between the two groups with respect to demographic and clinical characteristics. Fewer needle passes were required to obtain core specimens in the FNB group $(p<0.001)$. There were no differences in the proportion of histologic core $(11.8 \% \pm 19.5 \%$ vs $8.0 \% \pm 11.1 \%, p=0.376)$ or in the diagnostic accuracy $(80.6 \%$ vs $81.5 \%, p=0.935)$ between two groups. Conclusions: The proportion of histologic core and the diagnostic accuracy were comparable between the FNB and FNA groups. However, fewer needle passes were required to establish an accurate diagnosis in EUS-FNB. (Gut Liver 2017;11:559-566)

Key Words: Endosonography; Tissue sampling; Core tissue; Endoscopic ultrasound-guided fine needle aspiration; Endoscopic ultrasound-guided fine needle biopsy

\section{INTRODUCTION}

Since the introduction of endoscopic ultrasound-guided fine needle aspiration (EUS-FNA) in the early 1990s, it has become the standard procedure and has been widely used as a lessinvasive, reliable, and safe technique for cytopathologic diagnosis of solid mass lesions adjacent to the gastrointestinal tract (GIT). ${ }^{1-3}$ Although several previous studies demonstrated a favorable diagnostic sensitivity (64\% to 95\%) with high specificity (75\% to 100\%) and diagnostic accuracy (83\% to 95\%) in the diagnosis of pancreatic tumors, ${ }^{4-6}$ some limitations with a lower diagnostic yield have been reported in the evaluation of benign pancreatic lesions, neuroendocrine tumors, lymphadenopathies, and subepithelial lesions. ${ }^{7-9}$

Theoretically, the acquisition of a core tissue, which permits both histological examination and further immunohistochemical staining, might have advantages over aspiration of cytologic specimens. Various EUS-guided techniques have been explored to retrieve tissue specimens, including a conventional FNA needle and a Tru-Cut needle, with variable success and complication rates. ${ }^{7,8,10}$

Recently, EUS-guided fine needle aspiration biopsy (EUS-FNB) using a new core needle was developed to improve diagnostic accuracy by obtaining histologic core specimens. ${ }^{11,12}$ Many previous studies have revealed the advantages of a new core biopsy needle; less needle passes, more core tissue acquisitions, and higher diagnostic yields. ${ }^{13}$ However, there is no available data about the comparison of the histologic specimen itself obtained from a new core biopsy needle and a conventional needle. This study aimed to compare the histologic core in the samples obtained by EUS-FNB and EUS-FNA in patients with solid mass lesions.

\footnotetext{
Correspondence to: Chang-Min Cho

Department of Internal Medicine, Kyungpook National University Medical Center, 807 Hoguk-ro, Buk-gu, Daegu 41404, Korea

Tel: +82-53-200-2608, Fax: +82-53-200-2028, E-mail: cmcho@knu.ac.kr

Received on June 7, 2016. Revised on August 28, 2016. Accepted on September 17, 2016. Published online February 17, 2017 pISSN 1976-2283 eISSN 2005-1212 https://doi.org/10.5009/gnl16284

() This is an Open Access article distributed under the terms of the Creative Commons Attribution Non-Commercial License (http://creativecommons.org/licenses/by-nc/4.0) which permits unrestricted non-commercial use, distribution, and reproduction in any medium, provided the original work is properly cited.
} 


\section{MATERIALS AND METHODS}

\section{Patients}

Eligible patients referred to Kyungpook National University Medical Center for EUS-guided tissue sampling of solid mass lesions within the wall of or adjacent to the GIT were consecutively enrolled in this study. All solid masses were previously revealed by computed tomography or magnetic resonance imaging. Patients were excluded if a mass lesion was not found at EUS, if the mass had a cystic component of over $25 \%$, if the size of target lesion was less than $2 \mathrm{~cm}$ or if uncorrectable coagulopathy was present. This study was approved by the Institutional Review Board of Kyungpook National University Medical Center and registered on Clinical Research Information Service (KCT0001566). Written informed consent was obtained from all patients participating in this study.

\section{Endoscopic ultrasound examinations}

All procedures were performed according to the standardized protocols with left-lateral decubitus position and under moderate conscious sedation using meperidine, propofol, and/or midazolam. EUS was performed by a single experienced endosonographer (C.C.) who performs over 300 interventional cases per year. Following the identification of a target lesion using a radial scanning echoendoscope (GF-UE260; Olympus Medical Systems, Tokyo, Japan), EUS-guided tissue sampling was performed with a linear echoendoscope (GF-UCT240; Olympus Medical Systems).

\section{Technique for endoscopic ultrasound-guided tissue sampling}

The assignment of random numbers generated by computer were placed in sealed envelopes and opened by the nurse during the procedure if patients met the criteria for study inclusion. Patients were then randomized to undergo EUS-FNA using a 22-gauge conventional needle (EchoTip; Wilson-Cook Medical, Winston-Salem, NC, USA) or EUS-FNB using a 22-gauge core biopsy needle (EchoTip ProCore; Wilson-Cook Medical).

Once a target lesion was identified and the overlying vasculature was excluded using color Doppler flow, the needle was

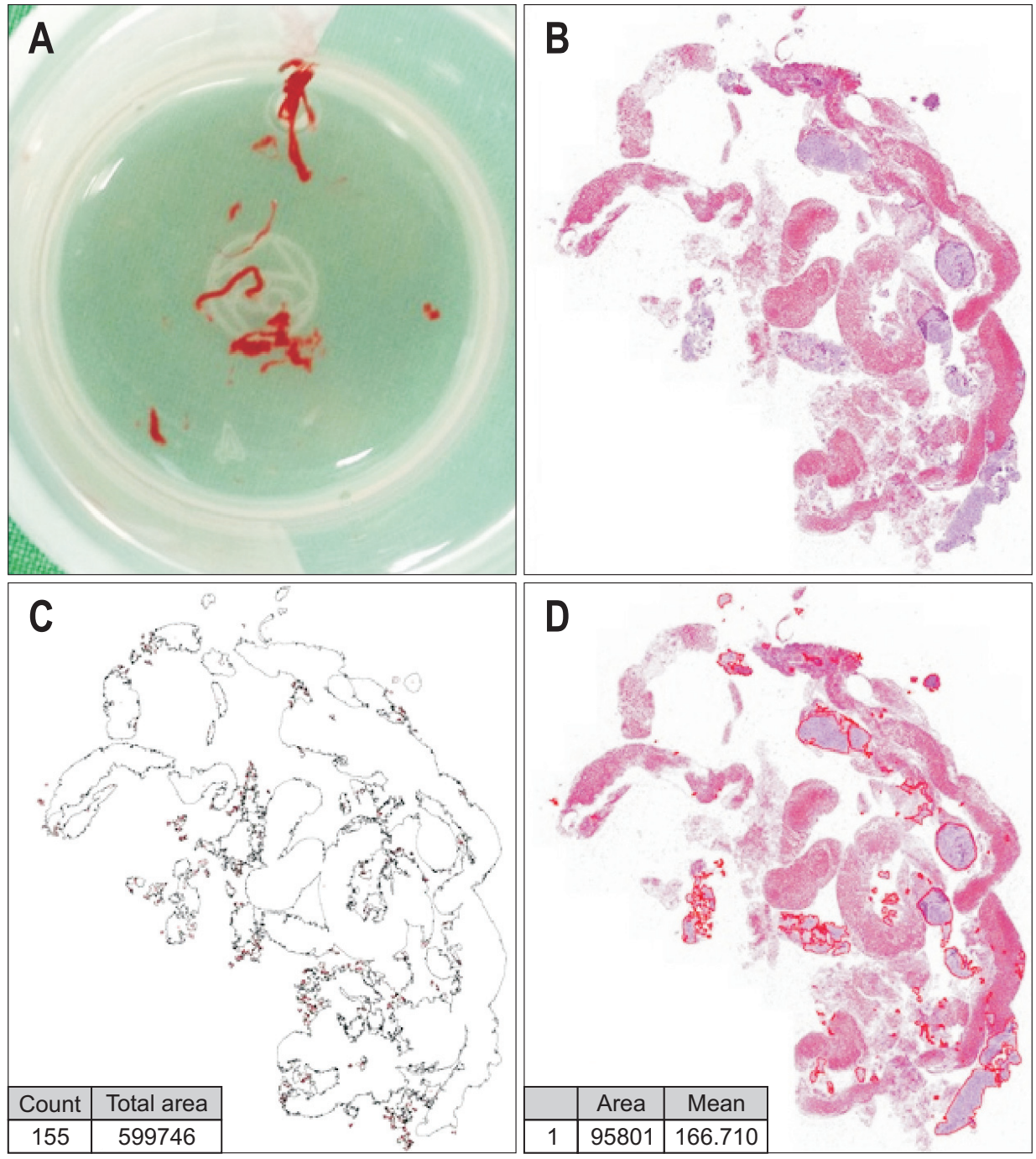

Fig. 1. Example of the measurement of overall and core portion areas. (A) Gross finding of an endoscopic ultrasound-guided fine needle aspiration specimen $(\times 40)$. (B) Hematoxylin and eosin staining of a histologic slide preparation. (C) Measurement of the overall area of the acquired specimen as 599,746 pixels $(\times 40)$. (D) Core tissue areas within redline measured 95,801 pixels. 
advanced through the instrument channel of the echoendoscope into the lesion. After puncturing the mass using the assigned needle, the stylet was slowly pulled by moving the needle toand-fro within the lesion. ${ }^{14}$ If a core specimen was not obtained using the slow pull technique, suction using a $10-\mathrm{mL}$ syringe was applied at the discretion of the performing endoscopist. The acquired tissue material was expelled into a formalin containing bottle by reinserting the stylet within the needle assembly. By examining the thread-like specimen, the endosonographer determined whether a visible core tissue has been obtained. Up to three needle passes were allowed until the core tissue specimen was acquired; no on-site cytologic examination was performed. If the initial three passes failed to retrieve an adequate specimen or in cases of technical failure (defined as malfunction of the needle during EUS-guided tissue sampling), the procedure was crossed over using the other needle in the study for up to three passes until sufficient diagnostic material was obtained.

\section{Histological assessment}

The core specimens obtained from both techniques were fixed in formalin, embedded in paraffin, sectioned, and stained with hematoxylin and eosin (H\&E) for histopathological evaluation. Additional immunohistochemical staining was performed for cases in which an adequate histological specimen was acquired. For histological assessment, a cytopathologist evaluated whether the tissue is sufficient to provide histologic diagnosis and undergo additional desired immunohistochemical studies. The cytopathologist was blinded to the needle type used during the procedure.

\section{Measurement of the total area and the core tissue area}

H\&E stained slides were scanned using a digital microscopy scanner of Panoramic MIDI (3DHistech, Budapest, Hungary) with a magnifying power of $\times 40$ and exported as a TIFF image for analyzing the total area and the histologic core area from the acquired specimen (Figs 1 and 2).
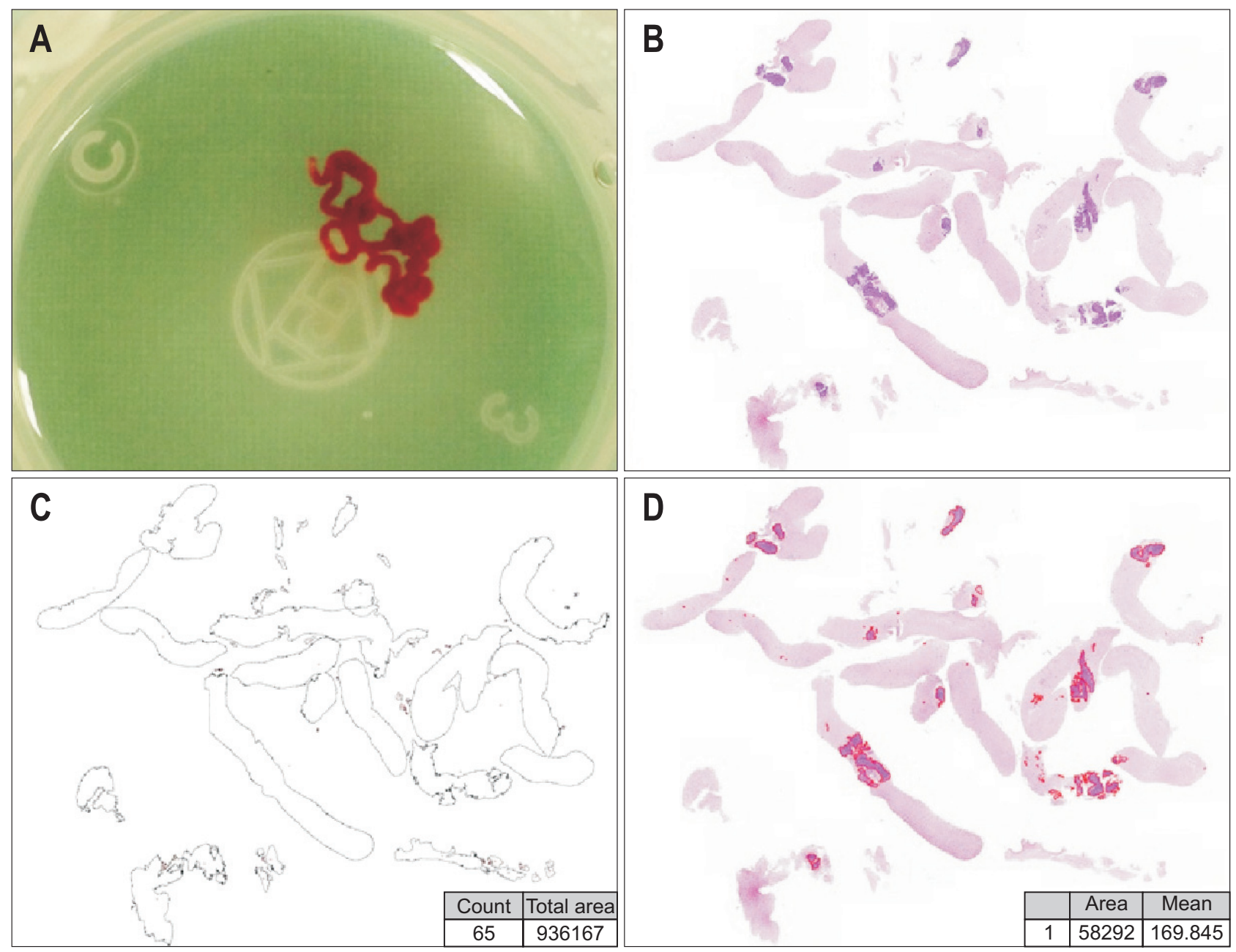

Fig. 2. Example of the measurement of overall and core portion areas. (A) Gross findings of the endoscopic ultrasound-guided fine needle biopsy specimen ( $\times 40)$. (B) Hematoxylin and eosin staining of the histologic slide preparation. (C) Measurement of the overall area of the acquired specimen as 936,167 pixels ( $\times 40)$. (D) Core tissue areas within redline measured 58,292 pixels. 
Using ImageJ software version 1.49f (National Institutes of Health, Bethesda, MD, USA), the exported TIFF images were used to measure and calculate both overall area and histologic core area. To measure total area in a scanned slide, the original scale was removed to unify the unit as pixel and the color image was converted to 8-bit gray scale. The image was adjusted to include all tissue materials by setting the minimum size as 50 and the maximum size as infinity. The total area was automatically calculated as pixels. Histologic core was defined as an architecturally correct piece of tissue sufficient for histologic evaluation. The area of the histologic core was measured using the freehand selection tool by a single cytopathologist (J.J.). The portion of histologic core was calculated dividing the histologic core area by the overall area and was then expressed as a percentage. The tutorials and examples for area measurements and particle counting were described in the web homepage of ImageJ (https://imagej.nih.gov/ij/docs/pdfs/examples.pdf).

\section{Outcome parameters}

The primary objective in this analysis was to compare the areas of the overall specimen and histologic core acquired by both techniques. The secondary objective was to compare the median number of passes required to obtain a core specimen (visible core), the technical success rate, diagnostic accuracy, and complication rates.

We defined the diagnostic accuracy as the sum of true-positive and true-negative results divided by the total number of specimens. All patients were followed for at least 1 year. Final diagnoses were made by either one of the following methods: (1) the histological assessment from surgically resected specimen; (2) the combined assessment of the EUS-FNA and EUSFNB samples for the clinical/imaging findings compatible with malignant or benign diseases; and (3) negative results of EUSFNA or EUS-FNB and no evidence of malignancy on clinical/ imaging follow-up of at least 1 year.

We defined adverse events as any postprocedure event attributable to EUS-FNA or EUS-FNB. All patients were hospitalized for at least 1 day after the procedure, and were observed for immediate adverse events. To monitor delayed adverse events, patients were interviewed by a routine telephone contact 1 week and 30 days after the procedure.

\section{Statistical analyses}

Two-tailed sample size calculation was performed with a type I error $(\alpha)$ of 0.05 and a power of $80 \%$ for detecting a difference in the proportion of histologic core tissue between the EUSFNA and EUS-FNB groups. Due to the lack of previous results for comparison, we referred to our own data. In the pilot trial, the average percentage of histologic core proportion from the acquired sample from EUS-FNA procedure was 10\%. Under the primary study hypothesis that EUS-FNB would provide more core tissue than EUS-FNA, we further assumed that EUS-FNB allowed a histologic core portion of $20 \%$ from the samples. The calculation yielded a target sample size of 29 for each cohort.

Categorical data were expressed as frequencies and proportions and compared using chi-square test or Fisher exact test as indicated. Continuous data were summarized as means with standard deviation (SD) and compared with the two-sample ttest, or the Wilcoxon rank-sum test. All statistical analyses were compiled using SPSS software version 18.0 (SPSS Inc., Chicago, IL, USA), with results considered significant at p-values of less than 0.05 .

\section{RESULTS}

\section{Patient demographics and characteristics}

Between April 2013 and May 2014, a total of 58 patients were enrolled and randomized for the study. Their demographic details and lesion characteristics are presented in Table 1. No

Table 1. Baseline Characteristics between the Endoscopic UltrasoundGuided Fine Needle Biopsy and Endoscopic Ultrasound-Guided Fine Needle Aspiration Groups

\begin{tabular}{|c|c|c|c|}
\hline Variable & $\begin{array}{c}\text { EUS-FNB } \\
(\mathrm{n}=29)\end{array}$ & $\begin{array}{c}\text { EUS-FNA } \\
(\mathrm{n}=29)\end{array}$ & $\mathrm{p}$-value \\
\hline Sex, male:female & $22: 7$ & $18: 11$ & 0.390 \\
\hline Age, yr & $66(36-81)$ & $69(26-85)$ & 0.534 \\
\hline Site & & & 0.927 \\
\hline Pancreas & 8 & 8 & \\
\hline Lymph node & 6 & 8 & \\
\hline \multicolumn{4}{|l|}{ Subepithelial lesions } \\
\hline Esophagus & 0 & 1 & \\
\hline Stomach & 6 & 3 & \\
\hline Small bowel & 1 & 1 & \\
\hline Rectum & 1 & 1 & \\
\hline \multicolumn{4}{|l|}{ Others } \\
\hline Liver & 3 & 4 & \\
\hline Retroperitoneum & 2 & 2 & \\
\hline Bile duct & 1 & 1 & \\
\hline Adrenal gland & 1 & 0 & \\
\hline \multicolumn{4}{|l|}{ Size, mm } \\
\hline Longest diameter & $37.5 \pm 20.6$ & $44.3 \pm 32.3$ & 0.348 \\
\hline Shortest diameter & $30.0 \pm 16.7$ & $33.8 \pm 23.3$ & 0.471 \\
\hline Approach & & & 0.993 \\
\hline Transesophageal & 3 & 3 & \\
\hline Transgastric & 17 & 16 & \\
\hline Transduodenal & 8 & 9 & \\
\hline Transrectal & 1 & 1 & \\
\hline No. of needle passes & $1(1-3)$ & $2(1-3)$ & $<0.001$ \\
\hline
\end{tabular}

Data are presented as number, median (range), or mean \pm SD. EUS-FNB, endoscopic ultrasound-guided fine needle biopsy; EUSFNA, endoscopic ultrasound-guided fine needle aspiration. 


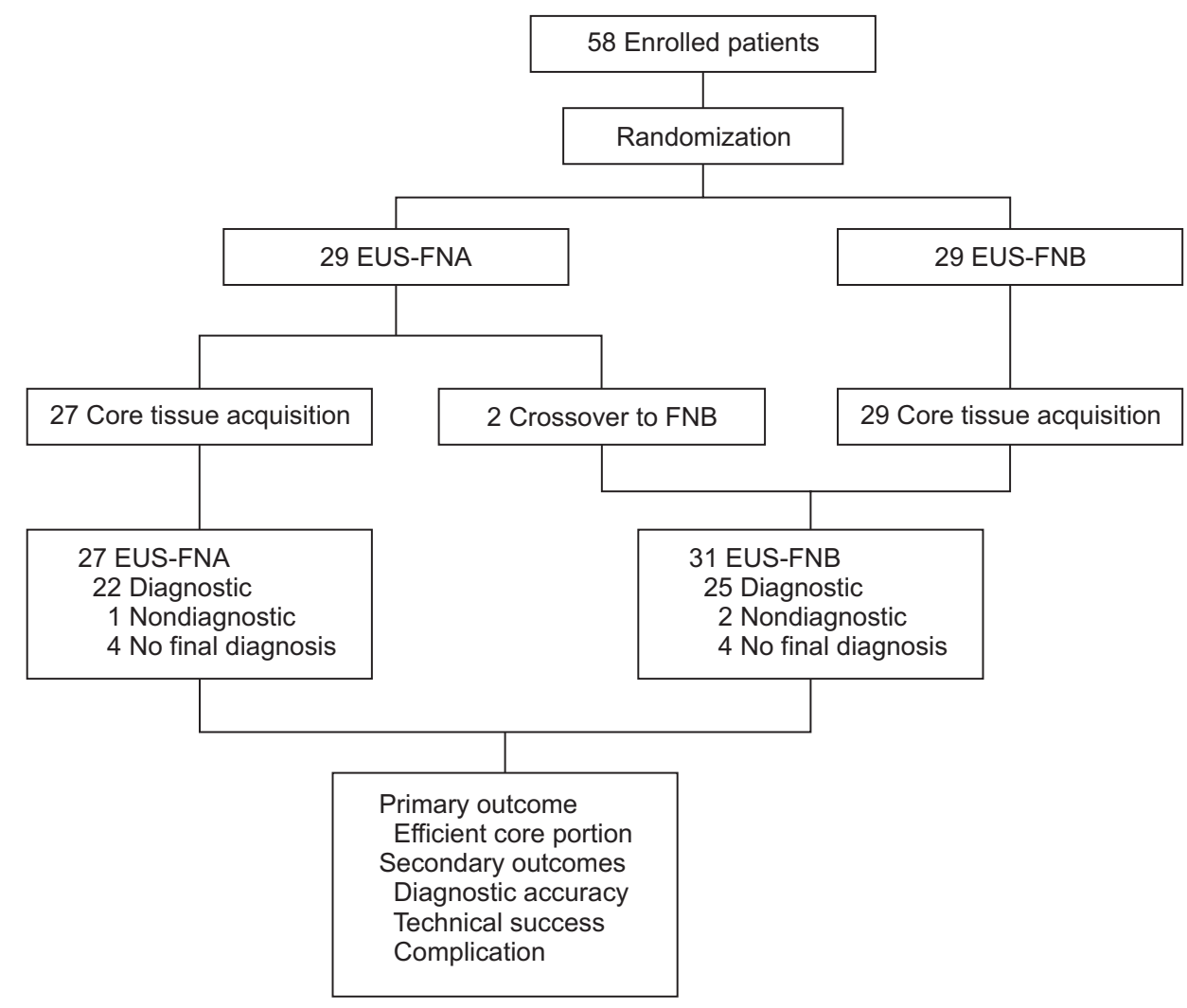

Fig. 3. Flow diagram of the study protocol.

EUS-FNA, endoscopic ultrasoundguided fine needle aspiration; EUSFNB, endoscopic ultrasound-guided fine needle biopsy. significant differences in patient age, sex, location and size of the tumor were detected between the EUS-FNA and EUS-FNB groups. Fewer needle passes were performed in the EUS-FNB group compared to the EUS-FNA group (1 vs 2, p<0.001). Patient flow through the study protocol is summarized in Fig. 3. No technical failure occurred in either group. Using the initial assigned needle, a visible core acquisition failed in two patients of the EUS-FNA group; each case of esophageal subepithelial lesion and pancreatic mass. Visible core tissue was successfully obtained in all cases of EUS-FNB group. Finally, 31 cases of EUS-FNB and 27 cases of EUS-FNA were included and compared for the primary and secondary objectives as per protocol analysis.

The final diagnosis at each target site is shown in Table 2. The final diagnosis was based on surgical pathology in 13 patients (22.4\%), EUS-FNA in 18 patients (31.0\%), EUS-FNB in 17 patients (29.3\%), and clinical/imaging follow-up in two patients (3.4\%). Among the enrolled patients, final diagnosis was inconclusive due to the inadequate tissue specimen and loss of follow-up after EUS-guided tissue sampling in each four patients of the EUS-FNA and the EUS-FNB group.

\section{Comparison of total area and histologic core area of the two techniques}

The overall area (megapixels, mean \pm SD) of the histologic slide prepared from specimens obtained by the EUS-FNB and the EUS-FNA were $1.26 \pm 1.09$ and $1.73 \pm 1.84$, respectively, with no significant difference $(\mathrm{p}=0.228)$ observed. When excluding the bias for needle pass, the overall area per needle pass was similar for the two techniques $(0.93 \pm 0.77$ and $0.76 \pm 0.69$, respectively; $\mathrm{p}=0.370$ ).

There were no statistical differences in the histologic core area of the total needle pass and per needle pass between the two techniques (Table 3). The proportion of histologic core tissue in terms of total area was also not significantly different between the FNB and FNA groups ( $11.8 \%$ vs $8.0 \%, \mathrm{p}=0.376$ ).

\section{Diagnostic accuracy between EUS-FNB and EUS-FNA}

The overall diagnostic accuracies per protocol were $80.6 \%$ and $81.5 \%$ in the EUS-FNB $(\mathrm{n}=31)$ and EUS-FNA ( $\mathrm{n}=27)$ groups, respectively. No significant difference in diagnostic accuracy was noted between two techniques $(\mathrm{p}=0.935)$. Comparing the diagnostic accuracy by intention-to-treat analysis, there was no significant difference between EUS-FNB (23/29, 79.3\%) and EUS-FNA (22/29, 75.9\%) ( $p=1.0)$. When stratified according to the specimen acquisition site, the diagnostic accuracy was also not different between the two groups (Table 4). No adverse events were observed in all cases.

\section{DISCUSSION}

EUS-FNA has been established as a safe and useful procedure for obtaining tissue samples from the wall of or adjacent to the GIT, including those from the pancreas and the retroperitoneum. 
Table 2. Final Diagnosis According to the Target Site in Both Groups

\begin{tabular}{|c|c|c|}
\hline Target site & $\begin{array}{c}\text { EUS-FNB } \\
(\mathrm{n}=31)\end{array}$ & $\begin{array}{c}\text { EUS-FNA } \\
(\mathrm{n}=27)\end{array}$ \\
\hline \multicolumn{3}{|l|}{ Pancreas } \\
\hline Pancreatic ductal adenocarcinoma & 5 & 3 \\
\hline Neuroendocrine tumor & - & 1 \\
\hline Autoimmune pancreatitis & - & 1 \\
\hline Chronic pancreatitis & 1 & - \\
\hline Inflammatory pseudotumor & 1 & - \\
\hline No final diagnosis* & 2 & 2 \\
\hline \multicolumn{3}{|l|}{ Lymph node } \\
\hline Malignancy lymphadenopathy & 4 & 4 \\
\hline Tuberculous lymphadenopathy & 1 & 2 \\
\hline No final diagnosis* & 1 & 2 \\
\hline \multicolumn{3}{|l|}{ Subepithelial lesions } \\
\hline Gastrointestinal stromal tumor & 5 & 4 \\
\hline Gastric cancer & - & 1 \\
\hline Esophageal cancer & 1 & - \\
\hline Glomus tumor & 1 & - \\
\hline Leiomyoma & 1 & - \\
\hline Malignant lymphoma & 1 & - \\
\hline \multicolumn{3}{|l|}{ Others } \\
\hline Cholangiocarcinoma, extrahepatic & 1 & 1 \\
\hline Cholangiocarcinoma, intrahepatic & 2 & 2 \\
\hline Neurogenic tumor & 1 & 2 \\
\hline Malignant lymphoma & 1 & - \\
\hline Hepatocellular carcinoma & 1 & - \\
\hline Hepatic metastasis & - & 2 \\
\hline No final diagnosis* & 1 & - \\
\hline
\end{tabular}

Data are presented as number.

EUS-FNB, endoscopic ultrasound-guided fine needle biopsy; EUSFNA, endoscopic ultrasound-guided fine needle aspiration.

*No final diagnosis due to the inadequate specimen and the loss to follow-up after EUS-guided tissue sampling.

However, the EUS-FNA technique has several limitations. First, the cytologic specimen alone is insufficient to verify cellular arrangement and tissue architecture. ${ }^{15}$ Therefore, it has a limited value in the diagnosis of certain disease entities, including gastrointestinal stromal tumors, lymphomas, well-differentiated adenocarcinomas, or autoimmune pancreatitis. ${ }^{8,16,17}$ Second, the success of EUS-FNA is greatly influenced by the definition used for diagnostic cytology and other methodological factors, such as availability of on-site evaluation, number of needle passes, and size of the needle. ${ }^{18-21}$

Recently, a new FNB needle with the application of reversed bevel technology has been developed to overcome these limitations of the EUS-FNA technique and to improve diagnostic accuracy by obtaining histologic core samples. The EUS-FNB needle was believed to improve diagnostic performance by pro-
Table 3. Comparison of the Areas of Histologic Specimens Acquired by the Endoscopic Ultrasound-Guided Fine Needle Biopsy and Endoscopic Ultrasound-Guided Fine Needle Aspiration Techniques

\begin{tabular}{lccc}
\hline & $\begin{array}{c}\text { EUS-FNB } \\
(\mathrm{n}=31)\end{array}$ & $\begin{array}{c}\text { EUS-FNA } \\
(\mathrm{n}=27)\end{array}$ & $\mathrm{p}$-value \\
\hline Overall area, megapixels & & & \\
$\quad$ Total needle pass & $1.26 \pm 1.09$ & $1.73 \pm 1.84$ & 0.228 \\
$\quad$ Per needle pass & $0.93 \pm 0.77$ & $0.76 \pm 0.69$ & 0.370 \\
Core tissue area, megapixels & & & \\
$\quad$ Total needle pass & $0.10 \pm 0.22$ & $0.09 \pm 0.12$ & 0.925 \\
$\quad$ Per needle pass & $0.06 \pm 0.11$ & $0.04 \pm 0.06$ & 0.595 \\
Efficient core portion, \%* & $11.8 \pm 19.5$ & $8.0 \pm 11.1$ & 0.376 \\
\hline
\end{tabular}

Data are presented as mean \pm SD.

EUS-FNB, endoscopic ultrasound-guided fine needle biopsy; EUSFNA, endoscopic ultrasound-guided fine needle aspiration.

*Expressed as percentage of core tissue area divided by overall area.

Table 4. Diagnostic Accuracy of the Endoscopic Ultrasound-Guided Fine Needle Biopsy and Endoscopic Ultrasound-Guided Fine Needle Aspiration Techniques

\begin{tabular}{lccc}
\hline & $\begin{array}{c}\text { EUS-FNB } \\
(\mathrm{n}=31)\end{array}$ & $\begin{array}{c}\text { EUS-FNA } \\
(\mathrm{n}=27)\end{array}$ & p-value \\
\hline Overall & $25 / 31(80.6)$ & $22 / 27(81.5)$ & 0.935 \\
Pancreas & $5 / 9(55.6)$ & $4 / 7(57.1)$ & 0.949 \\
Subepithelial lesion & $9 / 9(100)$ & $5 / 5(100)$ & 0.999 \\
Lymph node & $5 / 6(83.3)$ & $6 / 8(75.0)$ & 0.707 \\
Others & $6 / 7(85.7)$ & $7 / 7(100)$ & 0.299 \\
\hline
\end{tabular}

Data are presented as number/total number (\%).

EUS-FNB, endoscopic ultrasound-guided fine needle biopsy; EUSFNA, endoscopic ultrasound-guided fine needle aspiration.

viding better histological diagnostic accuracy; however, a recent randomized prospective study did not show any differences in the diagnostic yield or technical performance of the core biopsy needle compared to the conventional needle. ${ }^{11,13}$ In a small randomized trial including a total of 56 patients, EUS-FNB had comparable diagnostic yield to that of FNA (89.3\% vs 100\%, $\mathrm{p}=0.236$ ) with a similar number of needle passes (1.61 vs 1.28 , $\mathrm{p}=0.209){ }^{11}$ Although the EUS-FNB successfully procured material for cytological assessment, it was unsatisfactory for acquiring core biopsy material for histology. The authors concluded that while EUS-FNB obtained adequate aspirates for cytology, histological sampling with the 22-gauge EUS-FNB needle was unsatisfactory.

Another prospective study also demonstrated comparable diagnostic accuracy between FNB and conventional FNA needles. They suggested similar amounts of core acquisition as a possible explanation for the comparable results between the FNB and FNA groups. ${ }^{13}$ Given that there is no data available comparing the histologic specimen itself obtained from a core biopsy needle and a conventional needle, we directly compared the pro- 
portion of histologic core obtained by the two techniques. Our study showed there was no significant difference in the percentage of histologic core portion obtained and the diagnostic accuracy between the two techniques. Therefore, we confirmed that the similar diagnostic accuracy of the two methods observed in previous studies could essentially be attributed to a comparable yield of histologic core specimen.

Despite the comparable yield of the histologic core specimen, the number of needle passes was significantly lower in the FNB group, which was compatible with previous reports. ${ }^{13,22}$ The reverse bevel might have allowed a greater possibility of obtaining a suitable histologic core sample. The advantages of a fewer number of needle passes includes lower costs and less complications, as well as a reduction in procedure duration and need for anesthesia. In our study, needle passes were permitted up to three times until a visible core was acquired. Once a visible core was obtained, no additional needle passes were performed. Considering the fewer needle passes in the EUS-FNB group and the comparable yield of histologic cores, an equivalent number of needles passes in the two groups may have enhanced the yield of the histologic core in the EUS-FNB group. Previous investigations showing that diagnostic sensitivity improved as the number of passes increased would support our suggestion of favoring the FNB needle. ${ }^{23}$

This study has several limitations. First, this study was conducted at a single center, and the results may not reflect practices performed at other institutions. Second, an endoscopist, not an on-site pathologist, determined appropriate sample sizes containing a visible core; however, this is the current practice in majority of the facilities. Third, cytology data were not compared in this study, despite the final diagnosis depending on cytology as well as histology results. The comparable diagnostic grade of the cytology classification achieved between the two groups may support the hypothesis that a similar diagnostic accuracy between the two groups could be attributed to the similar amount of histologic core specimen obtained. Finally, various types of solid masses were included in the present study, possibly leading to heterogeneity of the study population. However, this enrollment criterion is compatible with current practice, given the widespread indications of EUS-FNA or EUS-FNB currently available, including subepithelial tumors, retroperitoneal masses, and lymph node enlargement, as well as pancreatic tumors. $^{24}$

Despite these limitations, our study has several important strengths that outweigh its weaknesses. First, this is the first trial comparing the actual histologic core specimen obtained by FNB and FNA needles. Second, our study was performed with a prospective randomized design. Finally, a single experienced endoscopist performed all procedures; this minimized the possible bias and established standardization of the procedures.

In conclusion, as compared to EUS-FNA, EUS-FNB required fewer needle passes to establish a diagnosis for solid mass le- sions. However, once an adequate sample containing visible core was obtained, the amount or the proportion of histologic core and the diagnostic accuracy were comparable for the two techniques.

\section{CONFLICTS OF INTEREST}

No potential conflict of interest relevant to this article was reported.

\section{ACKNOWLEDGEMENTS}

This work was supported by a Biomedical Research Institute grant from the Kyungpook National University Hospital (2013).

\section{REFERENCES}

1. Chang KJ, Albers CG, Erickson RA, Butler JA, Wuerker RB, Lin F. Endoscopic ultrasound-guided fine needle aspiration of pancreatic carcinoma. Am J Gastroenterol 1994;89:263-266.

2. Vilmann P, Hancke S, Henriksen FW, Jacobsen GK. Endosonographically-guided fine needle aspiration biopsy of malignant lesions in the upper gastrointestinal tract. Endoscopy 1993;25:523527.

3. Wiersema MJ, Chak A, Wiersema LM. Mediastinal histoplasmosis: evaluation with endosonography and endoscopic fine-needle aspiration biopsy. Gastrointest Endosc 1994;40:78-81.

4. Hewitt MJ, McPhail MJ, Possamai L, Dhar A, Vlavianos P, Monahan KJ. EUS-guided FNA for diagnosis of solid pancreatic neoplasms: a meta-analysis. Gastrointest Endosc 2012;75:319-331.

5. Chen G, Liu S, Zhao Y, Dai M, Zhang T. Diagnostic accuracy of endoscopic ultrasound-guided fine-needle aspiration for pancreatic cancer: a meta-analysis. Pancreatology 2013;13:298-304.

6. Itoi T, Sofuni A, Itokawa F, Irisawa A, Khor CJ, Rerknimitr R. Current status of diagnostic endoscopic ultrasonography in the evaluation of pancreatic mass lesions. Dig Endosc 2011;23 Suppl 1:1721.

7. Wiersema MJ, Vilmann P, Giovannini M, Chang KJ, Wiersema LM. Endosonography-guided fine-needle aspiration biopsy: diagnostic accuracy and complication assessment. Gastroenterology 1997;112:1087-1095.

8. Ribeiro A, Vazquez-Sequeiros E, Wiersema LM, Wang KK, Clain JE, Wiersema MJ. EUS-guided fine-needle aspiration combined with flow cytometry and immunocytochemistry in the diagnosis of lymphoma. Gastrointest Endosc 2001;53:485-491.

9. Mesa H, Stelow EB, Stanley MW, Mallery S, Lai R, Bardales RH. Diagnosis of nonprimary pancreatic neoplasms by endoscopic ultrasound-guided fine-needle aspiration. Diagn Cytopathol 2004;31:313-318.

10. Levy MJ, Jondal ML, Clain J, Wiersema MJ. Preliminary experience with an EUS-guided trucut biopsy needle compared with EUS-guided FNA. Gastrointest Endosc 2003;57:101-106. 
11. Bang JY, Hebert-Magee S, Trevino J, Ramesh J, Varadarajulu S. Randomized trial comparing the 22-gauge aspiration and 22-gauge biopsy needles for EUS-guided sampling of solid pancreatic mass lesions. Gastrointest Endosc 2012;76:321-327.

12. Iglesias-Garcia J, Poley JW, Larghi A, et al. Feasibility and yield of a new EUS histology needle: results from a multicenter, pooled, cohort study. Gastrointest Endosc 2011;73:1189-1196.

13. Lee YN, Moon JH, Kim HK, et al. Core biopsy needle versus standard aspiration needle for endoscopic ultrasound-guided sampling of solid pancreatic masses: a randomized parallel-group study. Endoscopy 2014;46:1056-1062.

14. Nakai Y, Isayama H, Chang KJ, et al. Slow pull versus suction in endoscopic ultrasound-guided fine-needle aspiration of pancreatic solid masses. Dig Dis Sci 2014;59:1578-1585.

15. Wahnschaffe U, Ullrich R, Mayerle J, Lerch MM, Zeitz M, Faiss S. EUS-guided Trucut needle biopsies as first-line diagnostic method for patients with intestinal or extraintestinal mass lesions. Surg Endosc 2009;23:2351-2355.

16. Erickson RA, Sayage-Rabie L, Beissner RS. Factors predicting the number of EUS-guided fine-needle passes for diagnosis of pancreatic malignancies. Gastrointest Endosc 2000;51:184-190.

17. Levy MJ, Reddy RP, Wiersema MJ, et al. EUS-guided trucut biopsy in establishing autoimmune pancreatitis as the cause of obstructive jaundice. Gastrointest Endosc 2005;61:467-472.

18. Afify AM, al-Khafaji BM, Kim B, Scheiman JM. Endoscopic ultra- sound-guided fine needle aspiration of the pancreas: diagnostic utility and accuracy. Acta Cytol 2003;47:341-348.

19. Iglesias-Garcia J, Dominguez-Munoz JE, Abdulkader I, et al. Influence of on-site cytopathology evaluation on the diagnostic accuracy of endoscopic ultrasound-guided fine needle aspiration (EUS-FNA) of solid pancreatic masses. Am J Gastroenterol 2011;106:17051710.

20. Turner BG, Cizginer S, Agarwal D, Yang J, Pitman MB, Brugge WR. Diagnosis of pancreatic neoplasia with EUS and FNA: a report of accuracy. Gastrointest Endosc 2010;71:91-98.

21. Song TJ, Kim JH, Lee SS, et al. The prospective randomized, controlled trial of endoscopic ultrasound-guided fine-needle aspiration using $22 \mathrm{G}$ and $19 \mathrm{G}$ aspiration needles for solid pancreatic or peripancreatic masses. Am J Gastroenterol 2010;105:1739-1745.

22. Hucl T, Wee E, Anuradha S, et al. Feasibility and efficiency of a new $22 \mathrm{G}$ core needle: a prospective comparison study. Endoscopy 2013;45:792-798.

23. Iwashita T, Nakai Y, Samarasena JB, et al. High single-pass diagnostic yield of a new 25-gauge core biopsy needle for EUSguided FNA biopsy in solid pancreatic lesions. Gastrointest Endosc 2013;77:909-915.

24. Noh KW, Woodward TA, Raimondo M, et al. Changing trends in endosonography: linear imaging and tissue are increasingly the issue. Dig Dis Sci 2007;52:1014-1018. 